\title{
Material Identifier with Attribute Identifier
}

National Cancer Institute

\section{Source}

National Cancer Institute. Material Identifier with Attribute Identifier. NCI Thesaurus.

Code C93862.

A unique symbol that establishes identity of the material. 